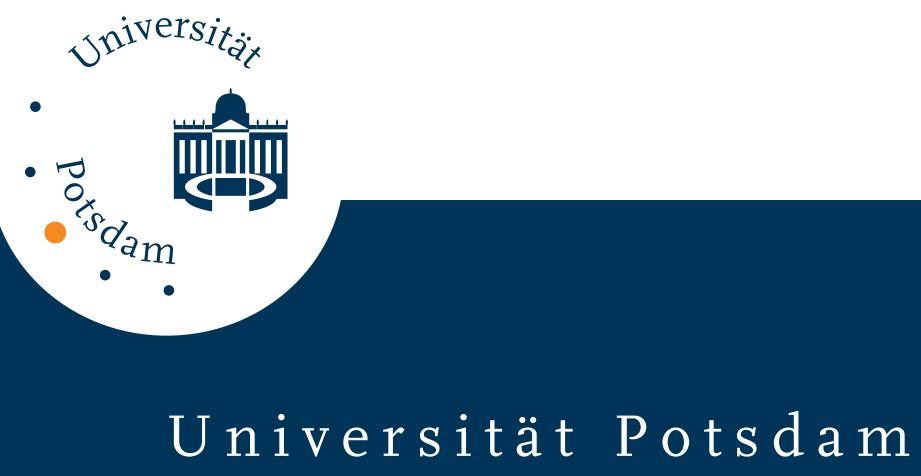

Reinhold Kliegl, Jacqui Smith, Jutta Hechhausen; Paul B. Bates

\title{
Mnemonic training for the acquisition of skilled digit memory
}

first published in:

Cognition and Instruction 4 (1987) 4, p. 203-223, ISSN 1532-690X, DOI

10.1207/s1532690xci0404_1

Postprint published at the Institutional Repository of the Potsdam University:

In: Postprints der Universität Potsdam

Humanwissenschaftliche Reihe ; 148

http://opus.kobv.de/ubp/volltexte/2009/4025/

http://nbn-resolving.de/urn:nbn:de:kobv:517-opus-40252

Postprints der Universität Potsdam

Humanwissenschaftliche Reihe ; 148 


\title{
Mnemonic Training for the Acquisition of Skilled Digit Memory
}

\author{
Reinhold Kliegl, Jacqui Smith, \\ Jutta Heckhausen, and Paul B. Baltes \\ Max Planck Institute for Human Development and Education \\ Berlin, Federal Republic of Germany
}

\begin{abstract}
This article outlines a research strategy for investigating, in a laboratory setting, the acquisition and the "limits" of a cognitive skill. Expert digit memory is used as an illustration. Two participants with initial average digitand word-span memory were trained to memorize and reproduce strings of $\mathbf{8 0}$ to 90 digits presented at 10 - to $1-\mathrm{sec}$ rates. The instruction and training program, based on a theory of skilled memory, focused on three components: (a) acquisition of a mnemonic system (i.e., recoding digits into historical dates or concrete nouns), (b) use of a long-term memory retrieval structure (i.e., instruction in the Method of Loci), and (c) improvement in processing speed. After 86 experimental sessions, one participant recalled 90 random digits presented at a 1-sec rate. The digits were, however, constrained to be compatible with the participant's historical knowledge. The second participant recalled 80 random digits presented at a 5 -sec rate after 70 sessions. Speed of encoding and retrieval processing was the only component that required extensive practice for skilled digit-memory acquisition.
\end{abstract}

To date, only a few studies have monitored the acquisition of complex cognitive expertise in a laboratory setting (e.g., Chase \& Ericsson, 1981; Neves \& Anderson, 1981). Although these studies monitored participants' performance changes, the participant rather than the experimenter controlled the development of the skill. Of course, this is also a problem in studies of real-life experts (Chi, Glaser, \& Rees, 1982). To test budding theories of cognitive learning, however, experimental control over knowledge accumulation, organization, and functioning seems desirable. In this article, we propose that systematic instruction in a cognitive skill provides

Requests for reprints should be sent to Reinhold Kliegl, Max Planck Institute for Human Development and Education, Lentzeallee 94, D-1000 Berlin 33, Federal Republic of Germany. 
a context for such control and for a better understanding of skilled performance than is possible using the traditional path of a posteriori "decompositional" analysis (see also Kliegl \& Baltes, 1987).

To illustrate our argument, we selected serial digit recall as an exemplar of cognitive expertise. Serial digit recall is typically characterized by performance levels of about seven digits, a narrow range of individual differences among adults (seven digits plus or minus two), and a strong resistance to improvement with simple practice (Miller, 1956). In contrast, most professional mnemonists perform this task at a much higher level (cf. Ericsson, 1985; Gordon, Valentine, \& Wilding, 1984; Hunt \& Love, 1972; Luria, 1968).

Two young adults participated in training programs designed to synthesize expert-like digit memory. Our instructional programs were modeled, in part, as analogies to models and descriptions of self-taught expertises available in the literature. Two detailed (observational) studies of expert digit memory were particularly relevant for our design: (a) a description of the Japanese mnemonist Ishihara (Susukita, 1933, 1934) and (b) a report of two long-distance runners who primarily used their knowledge of running times to code digits (Chase \& Ericsson, 1981, 1982; Ericsson, Chase, \& Faloon, 1980). These two studies (outlined later) provided the background for our choice of three components critical to expert performance: an encoding mnemonic, a knowledge-based retrieval structure, and processing speed.

\section{TWO EXAMPLES OF EXPERT DIGIT MEMORY}

Both Ishihara and the two runners observed by Chase and Ericsson integrated specific declarative and procedural knowledge to achieve their digit-memory expertise. Ishihara recoded subsets of two-digit to five-digit sequences into nouns using a digit-to-phoneme conversion scheme. In addition, Ishihara used a variety of multiple-use pegs or mental maps (similar to the Method of Loci) to encode the sequence of nouns resulting from recoding digits. He relied on personal knowledge to generate vivid images linking these mental map locations with the nouns. The particular map he used and the kind of digit-to-phoneme conversion depended on the digits he was asked to encode. In one trial, Ishihara recalled $97 \%$ of 2,400 digits he had studied for $6 \mathrm{hr}$ (i.e., about $9 \mathrm{sec} /$ digit).

Chase and Ericsson $(1981,1982)$ observed two young adults with otherwise normal memory abilities who acquired digit spans of 82 and 68 in 264 and 286 sessions of laboratory practice. Prior to this study, the best performances on digit-span tasks with fast presentation rates were around 20 digits and were typically displayed by mental calculators or professional 
mnemonists (Ericsson, 1985). These two participants generally chunked digits into running times and then created long-term memory retrieval structures by means of chaining and hierarchical ranking. The last digits of a trial were rehearsed in short-term memory.

\section{SKILLED MEMORY THEORY}

Based on a posteriori analyses of their two participants' performances, Chase and Ericsson (1981; Ericsson, 1985) formulated a model of memory skill that specified three theoretical components prerequisite for expert digit span: (a) a mnemonic system for recoding two, three, or four consecutive digits into meaningful chunks such as running times; (b) an effective and flexible long-term memory retrieval structure within which the recoded digits were organized in a multilevel tree structure; and (c) practice-related improvement in encoding and retrieval speed. This theory accounts for their participants' performance and is compatible with that of other mnemonists (Ericsson, 1985).

From the perspective of memory skill development, however, it would be important to determine whether these three components differ in acquisition difficulty. Research using mnemonic devices or recoding schemes suggests that normal individuals should readily acquire the first two components (e.g., Morris \& Greer, 1984; Slak, 1970). Ready improvement in recall of long lists of unrelated items associated with the use of various retrieval structures, such as the Method of Loci, is also well known (cf. Bellezza, 1981; Bower, 1970).

The third component, encoding and retrieval speed, could thus prove to be the critical characteristic for distinguishing expert from novice performance. Bugelski (1970; see also Bugelski, Kidd, \& Segman, 1968) concluded that a limitation of mnemonic tools such as the Method of Loci is the minimum of $4 \mathrm{sec}$ of encoding time required to fix a chunk in long-term memory even for persons with extensive practice. Simon's (1986) estimate for this operation is $8 \mathrm{sec} / \mathrm{chunk}$.

\section{TWO EXEMPLAR MODELS OF EXPERT DIGIT MEMORY}

Two models of expert digit memory were developed to illustrate different paths to expert performance: (a) the History-Dates model and (b) the Digit-Noun model. The development of these models was based on Chase and Ericsson's (1981) theory and on reports of professional mnemonists (Gordon et al., 1984; Hunt \& Love, 1972; Susukita, 1933). 
Both models used one mnemonic encoding and retrieval strategy, the Method of Loci (cf. Bower, 1970; Volkmann, 1929; Yates, 1966). The Method of Loci requires knowledge of an invariant mental map of locations: Each location in this map is used as an encoding peg and retrieval cue for information to be remembered. In our study, a series of 30 or 40 Berlin landmarks was used.

The two models of expert digit memory differed as to the kind of digit recoding the participants were instructed to use. In the History-Dates model, in addition to the Method of Loci, a participant is taught to select historical events and their associated dates. To encode sequences of digits according to this model, the participant recodes successive digit triplets into dates for historical events by mentally prefixing the triplet with a " 1 ." To memorize a sequence of triplets, one forms images involving events and Berlin landmarks of corresponding serial position (see Table 1 for illustration).

In the Digit-Noun model, in addition to the Method of Loci, the participant learned fixed associations between all possible digit numbers $(00$ to 99 ) and 100 concrete nouns. The digits corresponded to consonants and their sequential position in nouns following the Figure Alphabet mnemonic (Gordon et al., 1984; James, 1890; Lorayne \& Lucas, 1974; Morris \& Greer, 1984). The participant using the Digit-Noun model would alternately (a) recode consecutive digit pairs into concrete nouns and (b) use the Method of Loci to encode the sequence of nouns. Table 1 gives concrete examples for each of the two models.

TABLE 1

Illustration of History-Dates and Digit-Noun Models

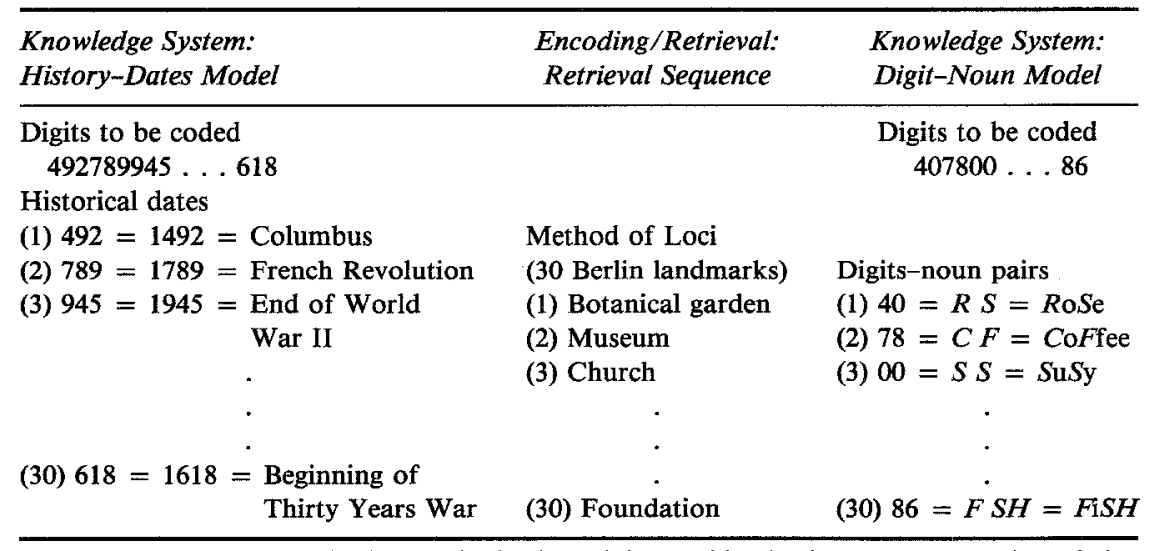

Notes. In each model, the Method of Loci is combined with one of two knowledge systems. In the History-Dates model, using digit triplets, 1,000 dates (000 to 999) would be necessary to encode all possible digit triplets. The Digit-Noun model, using digit pairs, provides a match to all random sequences based on 100 nouns. 
The models determined the components of two structured training programs. Two participants, one in each program, were systematically trained in the three central model components. They acquired a recoding mnemonic and a retrieval strategy, then practiced integrating these tools. Training interventions were set up to facilitate the final component, improving processing speed.

\section{METHOD}

\section{Participants}

Two males, ages 23 and 19 years, participated in the study. They were students who had no prior experience in the use of mnemonic techniques. They were paid 20 Deutsche marks (about 10 U.S. dollars) for each session.

One participant (SP) was instructed according to the History-Dates model. He completed 86 sessions in 14 months. Aside from two breaks during summer ( 2 months) and winter (1 month), hourly sessions were scheduled four times a week during the first 2 months before a summer break and twice a week thereafter. During the final 5 months, SP also worked about $12 \mathrm{hr} /$ week as a tutor for the Method of Loci in a training program involving other participants (Kliegl, Smith, \& Baltes, 1988).

A second participant (BB) was instructed according to the Digit-Noun model. He was assessed on baseline digit-span and instructed in the Method of Loci in February and March 1984 (14 sessions). He rejoined the project in March 1985 as a tutor for the Method of Loci, similar to participant SP. At this time, BB also resumed work as a study participant and completed 70 sessions in 5 months.

\section{Materials and Procedures for Components in the Training Program}

Apparatus. APPLE IIe personal computers were used for training and testing. Stimuli were presented on a monitor with a green display on black background. Participants entered their responses on a numeric keyboard.

Digit-span task. Baseline measures were taken on auditory and visual digit span at a presentation rate of $1.5 \mathrm{sec} /$ digit. Measures were also obtained for visually presented digits at a self-paced rate. In each condition, 20 trials were administered using a correct-one-up, wrong-one-down procedure across trials. The list length closest to a 50\% success rate was used as an estimate of memory span. 
Method of Loci. The Method of Loci required (a) overlearning a series of Berlin (West) landmarks and (b) forming associations between the loci and the input stimuli used in a given model (e.g., words or historical events). Instruction in the mnemonic strategy was similar to the one recommended by Bower (1970). Instruction began with a "sightseeing tour" of Berlin during which participants visited the 30 (participant SP) or 40 (participant BB) selected Berlin landmarks. They were given the ordered list of these landmarks and were asked to memorize this trip around Berlin. In the next session, when they could recite this list without error, participants were informed about the use of this landmark list as a memory tool.

Specifically, they were told to mentally visit the landmarks, always in a fixed sequence, and to generate funny or bizarre mental images or stories linking the item to be remembered and the landmark. In early sessions, they were asked to describe their images; the tutor (one of the authors or a research assistant) would evaluate the images and occasionally offer suggestions for improvement.

Training in the use of the Method of Loci began with word lists generated randomly from a pool of 1,560 concrete nouns extracted from a German dictionary. Prior to learning the system of loci, baseline measures of serial word recall had been taken. Participants were serially presented a list of words on the computer monitor. Immediately after the presentation, they attempted to recall the words and write them on an answer sheet. Scores were based on number of words recalled in correct serial position. The same procedure was used in training the Method of Loci.

History knowledge for History-Dates model (participant SP only). Short stories about 100 historical events were prepared as background material. Ten events were taken from each century between the years 1000 and 1799,10 events were taken from the periods of 800 to 850 and 950 to 1000 , and 10 events were taken from the period of 1850 to 1950 . The purpose of this was to sample representatively from all triplets between 000 and 999.

The 100 historical events were divided into four sets of 25. Participant SP learned each set in computer-administered test-and-study cycles to $100 \%$ correct recall of dates. Headlines of historical events of a set were presented in a randomized order for testing. The participant was required to enter the appropriate date. After each answer, the computer gave feedback about the correctness of the response and provided the correct date. Between tests, he could study incorrectly answered items of a set.

Nouns for Digit-Noun model (participant BB on/y). A list of 100 nouns for concrete objects was prepared with each noun coding one digit-doublet from 00 to 99 . Nouns were selected under the constraint that 
their first and second consonant sounds conformed to the digit-consonant correspondence. The following digit-consonant pairs were used: $0=s ; 1=$ $d, t ; 2=n ; 3=m ; 4=r ; 5=l ; 6=s c h ; 7=k ; 8=f ; 9=b, p$. This correspondence is known as the Figure Alphabet because there are visual similarities between the consonants and the digits they stand for (Gordon et al., 1984; Lorayne \& Lucas, 1974). Table 1 shows concrete examples of this coding scheme. Participant BB memorized the list of nouns outside the experimental setting and was told that he could substitute other nouns provided they conformed to the coding scheme; he substituted 49 words.

Integration of model components. As displayed in Table 1, the History-Dates and Digit-Noun models were explained to participants SP and $\mathrm{BB}$, respectively. Because they had already been instructed in the Method of Loci, there was no problem generalizing the strategy for memorizing lists of words to historical events or nouns derived from digit triplets or digit doublets.

\section{Procedures for the Refinement of Expert Digit Memory}

Procedure for participant SP. From Session 9 onward, SP was progressively challenged to extend and speed up his memory skill by changes in task demands. The criterion for practice schedule progression was that SP recall 90 digits in correct order. The target of 90 digits was set as a function of the limit of available locations (i.e., 30 landmarks).

Changes in task demands involved (a) increasing the pool of historical dates from which the 30 triplets in each digit sequence were randomly selected with replacement (from a pool of 25 to 100) and (b) increasing presentation rate per digit at encoding (from self-paced to the target of a 1 -sec fixed rate). We expected SP to gradually adapt his use of the History-Dates model to cope with increased task difficulty. Table 2 provides an overview of the time frame and progressive experimental changes in the training schedule.

Procedure for participant BB. BB was instructed to combine the Method of Loci with his digit knowledge as specified in Table 1. He always attempted to recall 80 random digits without any external aids. Again, as with participant SP, progressive changes in training interventions were introduced in an attempt to facilitate the development of BB's memory skill, in particular a speeding-up of processing. For BB, the only parameter change was presentation rate. The criterion for increase in rate was 80 digits recalled in correct order. 
TABLE 2

Summary of Participant SP's Experimental Schedule for Digit Memory Using the History-Dates Model

\begin{tabular}{llccc}
\hline Date & Sessions & Trials $^{\mathbf{a}}$ & $\begin{array}{c}\text { Presentation } \\
\text { Rate }^{\mathbf{b}}\end{array}$ & $\begin{array}{c}\text { Pool Size } \\
\text { of }^{\text {Triplets }}\end{array}$ \\
\hline $7 / 84$ & 10 to 18 & 1 to 9 & Self-paced & 25 to 75 \\
$10-11 / 84$ & 27 to 39 & 13 to 17 & Self-paced & 50 to 100 \\
$11-12 / 84$ & 42 to 48 & 20 to 25 & 3 & 100 \\
$2-3 / 85$ & 54 to 59 & 27 to 38 & 2 & 25 \\
$3-5 / 85$ & 60 to 76 & 39 to 61 & 1 & 25 \\
$6 / 85$ & 77 to 86 & 62 to 71 & 3 & 100 \\
\hline
\end{tabular}

${ }^{\text {a }}$ See also Figure 1.

${ }^{b}$ Either self-paced or in sec/digit.

${ }^{c}$ Number of historical triplets from which triplets were selected with replacement for construction of digit strings.

\section{RESULTS AND DISCUSSION FOR HISTORY-DATES MODEL (PARTICIPANT SP)}

Results of SP's performance are organized in four sections. First, we report his pretraining and posttraining performance. Second, we describe his acquisition of each of the component skills (Method of Loci, historical knowledge, and digit memory). Third, results of his expertise refinement are given. Refinement occurred in response to changes in presentation rate: from self-paced to a 1-sec/digit fixed rate. Finally, we demonstrate SP's compliance with the History-Dates model by error analyses.

\section{Comparison of Pretraining and Posttraining Performance on Model Components}

SP's digit span was assessed prior to the training, twice during training (before and after a summer break), and after the training program. His initial span under fixed presentation rates of $1.5 \mathrm{sec} /$ digit was 7 digits both in auditory and visual presentation modes. At the end of the training program, his scores had increased to 10 digits. On the intermediate tests, his scores ranged from 7 to 9 digits.

In contrast to the marginal transfer to standard digit span, SP's serial recall of words improved substantially after instruction in the Method of Loci. At pretest, he recalled 3 and 2 words of 30 in correct serial positions (presentation rate was $10 \mathrm{sec} /$ word and $8 \mathrm{sec} /$ word, respectively). Following the training, SP recalled 30 and 25 words of 30 presented in 4-sec and 2-sec intervals, respectively.

At pretest, SP knew the dates of 5 of the selected 100 historical events. On the final test, he answered 98 questions correctly. 


\section{Acquisition of Model Components}

Training sessions in the acquisition phase consisted of up to three different components: SP performed tasks related to the acquisition of the Method of Loci and of historical knowledge; he also attempted to improve his digit span under uninstructed practice.

Acquisition of Method of Loci. Upon instruction in Session 4, SP was immediately able to recall 30 words in correct order under self-paced encoding conditions (average encoding time: $52 \mathrm{sec}$ ). In each of the following 3 sessions, a new list of $\mathbf{3 0}$ words was recalled in perfect serial order. The average encoding time per word decreased from $52 \mathrm{sec}$ to $35 \mathrm{sec}$, $16 \mathrm{sec}$, and $27 \mathrm{sec}$ without instruction to go faster.

Acquisition of historical knowledge. As with the Method of Loci, SP had no problem acquiring a knowledge base of 100 historical events. In Sessions 3 through 18 , he required $8,7,7$, and 8 trials to recall the 25 events in each of four sets without error. SP continued to test his historical knowledge mostly as preparation for attempting a long string of digits.

Integration of model components. SP was instructed to combine the Method of Loci with his historical knowledge as specified by the History-Dates model (see Table 1). On the first trial to use the specified model (Session 7), SP recalled 24 of 25 triplets (72 of 75 digits); on the second trial (Session 8), he recalled 25 triplets (75 digits) in correct order. Thus, acquisition of the skilled digit-memory procedure was immediate. The primary goal of the following sessions was to chart the refinement of this skill.

\section{Refinement of Skilled Digit Memory}

Strategy of data analysis. SP's recall of correct digit triplets in correct serial position was analyzed with multiple regression. Using his mean performance under self-paced encoding conditions as a baseline, parameters were specified to test for a significant drop in performance as a response to training interventions (i.e., changes in presentation rate) and to test for linear trends coding the improvement across trials within training conditions. Table 3 lists those parameters. Coefficients without parentheses were significant at $p<.05$.

In 9 of the 71 trials, SP terminated his encoding activities before half the digits had been presented. In these cases, then, recall was not a function of quality of encoding but of lack of encoding. In addition, 7 other trials contained a response pattern indicating that, either during encoding or 
TABLE 3

Ordinary-Least-Squares (OLS) and Maximum-Likelihood (ML) Estimates for Participant SP's Data

\begin{tabular}{lcc}
\hline Coefficient & $O L S$ & $M L$ \\
\hline Effect of experimental manipulation & & \\
Mean of self-paced condition & 29.3 & 29.1 \\
Change to 3-sec condition & -9.3 & -9.1 \\
Change to 2-sec condition & $(-1.4)$ & $(0.3)$ \\
$\quad$ Associated linear trend & $(.09)$ & $(-.31)$ \\
Change to 1-sec condition & -15.2 & -14.4 \\
$\quad$ Associated linear trend & .84 & .76 \\
Reversal to 3-sec condition & -5.5 & -4.2 \\
$\quad$ Associated linear trend & $(.60)$ & .42 \\
Time series parameters & & -.65 \\
First-order autoregression coefficient & - & -.54 \\
Second-order autoregression coefficient & - & \\
\hline
\end{tabular}

Notes. Effects of changes in experimental conditions were tested against self-paced trials as baseline. For example, in the 1-sec condition, performance was 15.2 triplets below the mean of the self-paced condition. $R^{2}=.75$ for complete OLS solution. Coefficients without parentheses were significant at $p<.05$. (See also Footnote 2.)

during recall, SP systematically displaced four or more triplets in a positive or negative direction. Such a pattern is likely to result from accidentally skipping a landmark either at encoding or at recall. Because performance on trials with systematic shifts (reflecting an inadequate use of the mental map) is qualitatively different from other trials, it was decided to exclude these trials from the main analysis. Finally, 7 trials with exploratory experimental conditions departing from the main schedule were excluded. ${ }^{1}$ The analysis, therefore, was based on 48 of the entire set of 71 trials. $^{2}$

Figure 1 displays SP's performance in response to training interventions devised to stimulate the further development of his digit memory skill. Details relating to each change in SP's performance are described below.

Self-paced presentation rate (Trials 1 to 17). As shown in Figure 1, under self-paced conditions SP was able to perform immediately according

\footnotetext{
${ }^{1}$ We were also interested in whether the participant would be able to move to a high score given a low starting value and using the correct-one-up and wrong-one-down procedure. In the first session with 20 trials, he moved from 5 to 16 digits. In a second session with 14 trials and starting with 12 digits, he increased the span to 20 .

${ }^{2}$ Because data were produced by a single participant, the assumption of uncorrelated errors could be violated. Inspection of autocorrelations and partial autocorrelations suggested that residuals followed a first- and second-order autoregressive process. To obtain valid statistical confidence intervals for parameter estimates, the model (augmented by two autoregressive parameters) was also tested in a time-series analysis. Maximum-likelihood estimates obtained in this analysis are displayed in Table 3 . These two analyses yielded very similar results.
} 


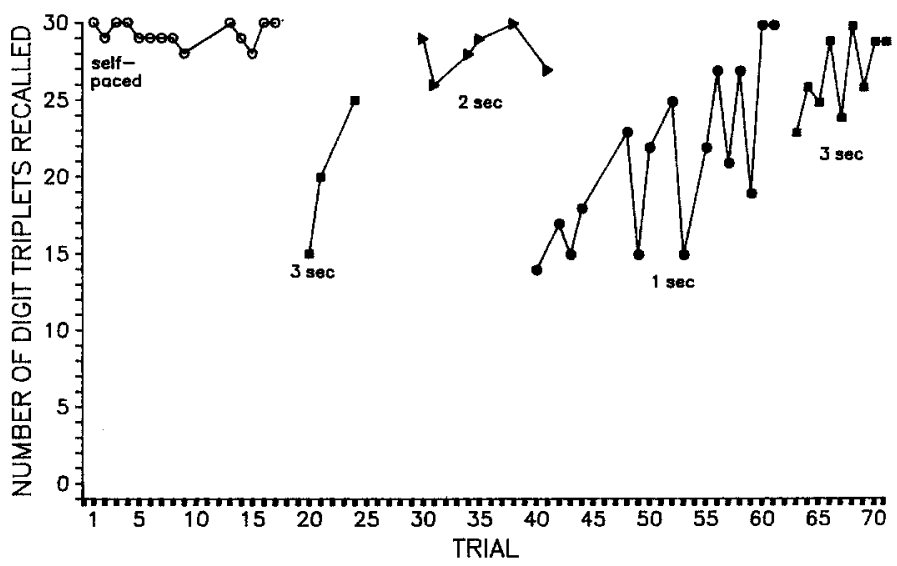

FIGURE 1 History-Dates model. SP's recall of correct digit triplets (maximum $=\mathbf{3 0}$ triplets or 90 digits) as a function of trial number and presentation rate.

to the History-Dates model at a close to perfect level. This sequence served as a baseline for later trials.

During self-paced baseline conditions, there was also a systematic decrease from an average of $23 \mathrm{sec}$ to around $11 \mathrm{sec}$ required for encoding single digits. This average encoding time was not evenly distributed across digits. As expected, SP quickly obtained digits forming a triplet and then spent a longer time encoding the event. Thus, the encoding time per historical date-chunk was on average $34 \mathrm{sec}$ in the last 6 self-paced trials. ${ }^{3}$ As indicated in Table 2, digit strings in this condition were constructed from triplet pools of sizes 25 to 100 . Because performance was uniformly high, pool size of historical dates obviously had no effect.

Three-sec rate and pool size of 100 triplets (Trials 20 to 25). In Trials 20 to 25 , SP attempted to memorize 90 digits presented at 3-sec intervals. According to SP's report, he was not able to create crisp images for the historical events at this fast speed. For this reason, he could not keep up his systematic encoding using the Method of Loci and did not complete 3 of 6 trials. Figure 1 shows his performance on the 3 completed trials. The decline in performance, even if measured only for completed trials, was significant (see Table 3).

Two-sec rate and pool size of 25 triplets (Trials 27 to 38). To assist SP in overcoming his problem with fast presentation rates, following a

\footnotetext{
${ }^{3}$ No response latencies for entering single digits were collected during recall. From observing SP, however, it was clear that he would recall a date, quickly type in the corresponding three digits, and then attempt to recall the next triplet. Similar observations were made when BB recalled digit doublets. In his case, there were clear breaks after each second digit.
} 
2-month winter break several sessions were spent encouraging SP to devise crisp images for the first set of 25 historical events. Following this substitution of complex historical events with simple concepts and 6 practice trials with fewer digits, SP performed 11 trials at a 2 -sec presentation rate. Four trials (including the first three) were terminated early; during another trial, a systematic displacement of digits occurred. Thus, only 6 trials (see Figure 1) were completed according to our stated criteria. On these completed trials, SP consistently recalled 26 or more triplets in correct order. Although SP completed only about $50 \%$ of the trials, similar to the previous condition, performance on completed trials with a small set of triplets at a faster presentation rate was always better compared to the previous condition and not significantly different from the self-paced baseline $(p>.05$; see Table 3$)$.

One-sec rate and pool size of 25 triplets (Trials 39 to 61). Starting in Session 60 (Trial 39 in Figure 1), 21 trials were performed with a 1-sec rate. One trial was not completed; systematic displacements occurred on 4 other trials.

As reflected in corresponding regression coefficients in Table 3, there was a significant drop in performance relative to the self-paced baseline as a consequence of this intervention. However, there was also a significant linear improvement within this condition $(p<.05$; see Table 3$)$. During the last trials of this sequence, SP correctly recalled 90 digits twice.

Three-sec rate and pool size of 100 triplets (Trials 77 to 86). Finally, as in the initial previous series of trials, digit sequences were composed from the original pool of 100 historical events and were presented at rates of $3 \mathrm{sec} /$ digit. The pool of triplets contained the 25 events for which special images had been formed plus 75 for which no special images had been formed. SP completed all but the first of 10 trials in this condition. Performance was significantly lower relative to self-paced baseline $(p<$ .05 ; see Table 3 ), but there was also no overlap in performance with SP's initial series of trials with a $3-\mathrm{sec}$ presentation rate.

\section{Error Analysis}

If SP worked with the History-Dates model, we would expect certain types of errors to occur more frequently than others. Five error types were discerned: (a) a forgotten triplet, (b) a misplaced triplet (i.e., a triplet recalled at a wrong landmark), (c) an incorrect triplet from the pool of history dates active during the trial, (d) an incorrect triplet from the pool not active during trial, and (e) a triplet that was not part of the pool of 100 . If SP performed according to the History-Dates model, the first three error 
types should be more frequent than the last two, because these last two error types do not involve model components.

We counted 292 errors in 1680 encoded triplets; thus, the overall error rate was $17 \%$. Of the 292 errors, $61 \%$ were forgotten, $16 \%$ were misplaced, and $23 \%$ were incorrect triplets from the pool active during the trial. There were no incorrect triplets from the pool not active during the trial. Finally, only 2 errors were not part of the pool of 100 triplets; most likely they were typographical errors (007 instead of $077 ; 357$ instead of 857). Thus, SP's error pattern was consistent with the expectation that he would rely on the sequence of locations and the historical knowledge specified when performing the memory task.

\section{Discussion}

SP readily acquired an expertise in digit memory when relying on the History-Dates model provided by the experimenter. Within a year and after 71 trials, he reached a level of competence quite comparable to that of professional mnemonists. SP displayed a stable level of performance with digits presented once per second. However, this performance level was confined to digit strings composed of a small pool (25 to 100) of triplets. When digit sequences could not be parsed into his special historical knowledge, his memory was only slightly better than his pretraining performance. This was reflected most directly in the small amount of transfer to the standard digit-span tests.

As in Chase and Ericsson's (1982) work, specialized knowledge appeared as a prerequisite for high performance with fast presentation rates. Consequently, recall of completely random digit strings according to the History-Dates model would require knowing 1,000 mutually exclusive historical dates (000 to 999$)$. It is unclear how long it would take to acquire this much factual knowledge and to use it at fast presentation rates. The results suggest, however, that of the three components Chase and Ericsson (1982) postulated as prerequisites of skilled memory, only improvement in encoding and retrieval speed requires a large amount of practice. Acquisition of a mnemonic system for recoding digits into imaginable chunks and acquisition of a long-term memory encoding and retrieval structure such as the Method of Loci were accomplished easily.

\section{RESULTS AND DISCUSSION FOR THE DIGIT-NOUN MODEL (PARTICIPANT BB)}

The Digit-Noun model was designed to provide an alternative model of engineering skilled digit memory. A possible disadvantage associated with 
the History-Dates model was that it would require the acquisition of knowledge for 1,000 triplets to simulate a "true" digit span. Therefore, a second pathway to expert digit memory, the Digit-Noun model, was designed to be compatible with any random sequence of digits. In this model, the participant had to acquire knowledge in the form of concrete nouns for 100 digit-doublets. To code a similar number of digits (i.e., 80), the mental map was extended to encompass 40 locations.

Participant BB was trained to use the Digit-Noun model to develop his skill in digit memory. Data for BB are presented in three parts: (a) effects of uninstructed practice on digit span, (b) a description of his skill acquisition and refinement in response to training interventions, and (c) an error analysis to demonstrate his use of the Digit-Noun model.

\section{Effects of Uninstructed Practice on Digit Span}

Prior to training, BB completed a total of 300 baseline trials of digit span. There were 20 trials in each block and one to four blocks per session. Digits were presented at a rate of $1.5 \mathrm{sec} /$ digit. On 159 trials, spans of 7,8 and 9 digits were attempted and $60 \%, 46 \%$, and $30 \%$, respectively, were correctly recalled. There was one correct recall each for 10-, 11-, and 12-digit spans. No improvement was noticeable in performance despite this large number of practice trials. Thus, BB's span was estimated at 8 digits.

\section{Acquisition of Skilled Digit Memory}

Acquisition of Method of Loci. In the first session, BB recalled in correct serial position 4 and 6 words from a list of 30 presented at rates of 10 and $4 \mathrm{sec} /$ word. In Sessions 5 through 12, he practiced the Method of Loci and correctly recalled 29 of 30 words at self-paced rates of 14, 13, and $12 \mathrm{sec} /$ word. Thus, BB had a normal memory span for words but was immediately able to successfully use the Method of Loci to recall a much larger number of words.

Integration of model components. Following 4 practice trials, during which BB had the list of nouns and the list of Berlin landmarks available for reference during encoding and retrieval, BB always attempted to recall 80 digits without any external aids. Figure 2 displays his performance after instruction in the Digit-Noun model across a 5-month period and shows his response to training interventions. Each trial corresponds to 1 session.

At first (Trials 1 to 7), BB determined the presentation rate of digits himself. As Figure 2 shows, BB (like SP) immediately performed at a very high level of accuracy. His average time for encoding digits decreased from 13.4 to $9.7 \mathrm{sec}$. 


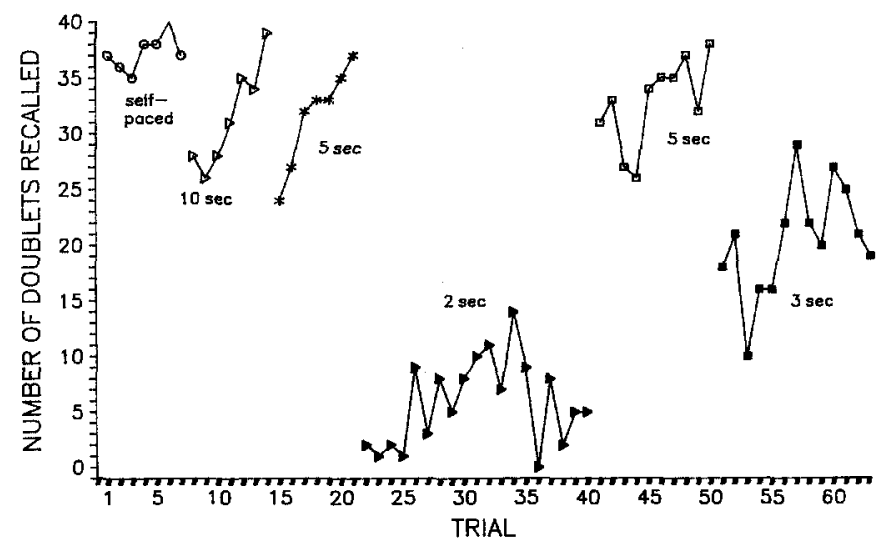

FIGURE 2 Digit-Noun model. BB's recall of correct digit doublets (maximum $=\mathbf{4 0}$ doublets or 80 digits) as a function of trial number and presentation rate.

\section{Refinement of Skilled Digit Memory}

On the remaining trials, BB's memory skill was tested under various fixed presentation rates. A complete account of his performance is displayed in Figure 2 . The data were fitted by a regression model using 12 parameters specifying the progressive interventions in BB's training program (see Table 4). The parameters estimated number of doublets recalled in the self-paced condition, BB's change in performance when a different presentation rate was introduced, and his subsequent (linear) improvement under this condition.

TABLE 4

Ordinary-Least-Squares (OLS) Estimates for Participant BB's Data

\begin{tabular}{lc}
\hline Coefficient & OLS \\
\hline Mean of self-paced condition & 37.2 \\
Change to 10-sec condition & -13.7 \\
Associated linear trend & 2.0 \\
Change to 5-sec condition & -13.7 \\
Associated linear trend & 2.0 \\
Change to 2-sec condition & -39.3 \\
Associated linear trend & 1.9 \\
Associated quadratic trend & -.08 \\
Reversal to 5-sec condition & -9.0 \\
Associated linear trend & .81 \\
Change to 3-sec condition & -20.9 \\
Associated linear trend & .59 \\
\hline
\end{tabular}

Notes. Changes were tested against self-paced trials as baseline. $R^{2}=.95$ for complete model. For all coefficients, $p<.05$. 
The $R$ for this model was $.97\left(R^{2}=.94\right)$. Table 4 summarizes coefficients and associated standard errors; all coefficients were significant $(p<.05)$. The bulk of the variance (i.e., 90\%) was due to changes in presentation rates. The linear trends within experimental conditions accounted for an additional $3 \%$ of the variance. Inspection of the performance profile also suggested a quadratic trend in the 2 -sec condition. A parameter coding this trend added an additional $1 \%$ of the variance. Both increments were significant $(p<.01)$. Because all data were obtained from one participant, the assumption of uncorrelated residuals was checked: There were no significant autocorrelations or partial autocorrelations in the residuals. Thus, statistics based on ordinary-least-squares estimates can be considered valid. ${ }^{4}$

Proceeding from a self-paced format to digit presentation at fixed rates of $10 \mathrm{sec}$ and $5 \mathrm{sec}$ (Trials 8 to 14 and Trials 15 to 21 , respectively) had similar effects: There was a significant immediate drop in performance and subsequent significant linear recovery. This is evident in Figure 2. When digits were presented at a 2 -sec rate, however, BB could not restore performance to previous levels (Trials 22 to 40 ). There was also a significant quadratic trend in this condition: An initial improvement was followed by later decline.

The breakdown in performance was so dramatic that we opted not to push for a 1-sec presentation rate. BB recalled an average of 5.8 digitdoublets at the 2 -sec rate. If short-term memory capacity is based on chunks, his performance was actually below the estimate of 8 obtained in pretraining practice of standard digit span. Therefore, we moved BB back a level in the training intervention schedule (5-sec presentation rate) to ascertain whether the drop in performance was related to encoding time. Performance was resumed significantly below baseline (Trial 41) but again showed fast linear improvement on the following trials (Trials 41 to 50 in Figure 2).

After BB had regained criterion performance ( 80 digits correct) at 5 $\mathrm{sec} /$ digit, he was now challenged by a presentation rate of $3 \mathrm{sec} /$ digit rather than $2 \mathrm{sec} /$ digit. With $3 \mathrm{sec} /$ digit, BB's performance again dropped significantly (Trial 51), but this time there was significant linear improvement on subsequent trials (Trials 51 to 63 in Figure 2).

In summary, BB's performance profile corresponded closely to the amount of time available during encoding: Performance was highest for self-paced, 10-sec, and 5-sec encoding rates; intermediate for $3 \mathrm{sec} / \mathrm{digit}$;

\footnotetext{
${ }^{4} \mathrm{As}$ is obvious from Table 4, several parameter estimates were almost identical. A more parsimonious model with seven parameters (specifying two instead of seven parameters to code change and linear trend in the 10-sec and two 5-sec conditions and the same trend for the 3-sec condition) resulted in $R=.96$, nearly identical to that for the 12-parameter model.
} 
and lowest for $2 \mathrm{sec} /$ digit. Thus, presentation rate manipulations identified conditions under which performance could be improved or depressed with and without subsequent recovery.

\section{A Test of Model Usage}

The regression analysis demonstrated reliable effects of task difficulty on performance and also significant improvement across trials with the exception of the fastest presentation rate. On the basis of this analysis alone, however, it cannot be decided whether BB performed according to the Digit-Noun model or whether he designed his own creative strategy. Moreover, his degree of compliance with the model could depend on the presentation rate. There are, however, two testable implications of using the Digit-Noun model:

1. Digits should be recalled in pairs, because converting digits to words involves two consecutive digits.

2. If the mental map of Berlin landmarks was used properly, the first digits of correct doublets should be more often in odd than in even serial positions (see also Footnote 3).

To test these predictions, we computed two scores of correct doublets for each trial. The scores were based (a) on the number of correct doublets starting in an odd serial position (as in Figure 1) and (b) on the number of correct doublets starting in an even serial position. If $\mathbf{B B}$ performed according to the Digit-Noun model, his odd score should exceed his even score.

In addition, a baseline score for correct doublets was estimated on the basis of the number of single digits correct. Obviously, for reasons of combination, the more single digits recalled, the higher the probability of correct doublets. Specifically, the probability of correct digit doublets is the square of the probability of correct single digits.

For the following analysis, the first and the last digit of a trial were ignored because they would only contribute to either the odd or the even score. Furthermore, perfectly recalled trials would by necessity lead to identical scores. Therefore, only trials with fewer than 72 (i.e., 90\%) digits recalled were included. Finally, we combined trials with self-paced, 10-sec, and 5 -sec rates in a category of slow presentation rates.

Means of odd, even, and estimated baseline scores are displayed as a function of presentation rate in Figure 3. As expected, odd scores were always higher than even scores. This effect was also significant in a mixed-model analysis of variance with presentation rate (three levels) as a between-trial variable and two contrasts (odd vs. even and even vs. baseline 


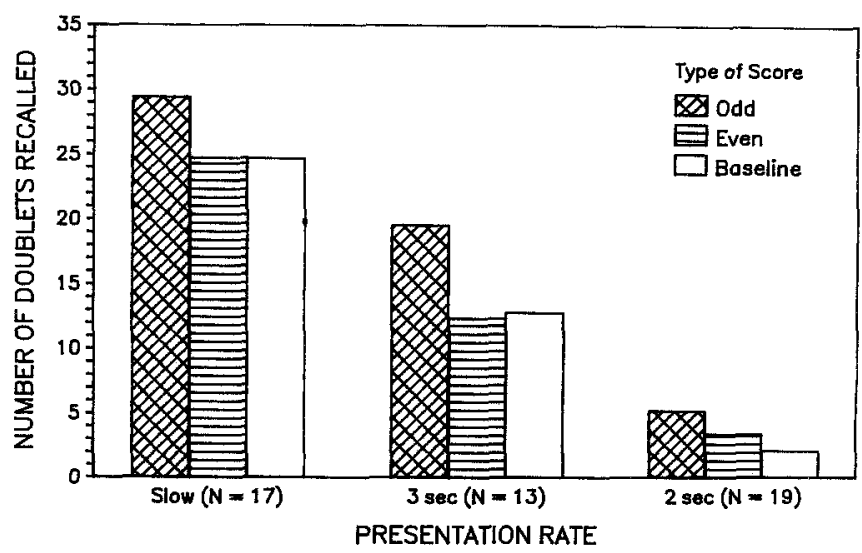

FIGURE 3 BB's recall of digit doublets as a function of presentation rate and three scoring criteria (see text).

scores) as within-trial variables, $F(1,46)=327.6, p<.01$. Also in line with expectations, the difference between even and baseline scores was not significant, $F(1,46)=2.0, p>.15$.

The contrast specifications interacted significantly with presentation rate. First, differences between odd and even scores were largest for the 3-sec rate and smallest for the 2 -sec rate, $F(2,46)=24.4, p<.01$. This interaction could, however, result from a statistical artifact: By statistical necessity the range of possible scores is largest for medium-difficulty conditions. Therefore, the 3-sec condition provided the most opportunities to observe differences between odd and even scores.

The second contrast indicated that even scores were significantly higher than the estimated baseline scores only under the fastest presentation rate, $F(2,46)=6.2, p<.01$. Perhaps with $2 \mathrm{sec} /$ digit encoding time, BB occasionally missed a digit and slipped into an even encoding. Thus, at a 2 -sec rate, BB failed to employ the Digit-Noun model effectively.

In conclusion, the pattern of results clearly agreed with a theoryconsistent model usage for presentation rates up to $3 \mathrm{sec} /$ digit. At the 2-sec rate, BB could not maintain his level of skill. Mnemonist-like performance was demonstrated, but only with moderate presentation rates.

\section{GENERAL DISCUSSION}

We have demonstrated two pathways to expert digit memory. High performance levels in terms of numbers of digits recalled were attained both in the History-Dates and Digit-Noun models. Participant SP displayed a consistent performance with lists of 90 digits at a 1 -sec rate in the 
History-Dates model. His skill was confined, however, to experimental conditions in which consecutive digit triplets could be converted to the dates for the historical events in his knowledge base. In the second case study, unconstrained digit lists were used on all trials. Participant BB recoded consecutive digit doublets into subsamples of an exhaustive set of 100 concrete nouns. For him, presentation rates faster than $3 \mathrm{sec} /$ digit appeared as a limiting factor of the skill. With 2-sec rates, performance was close to chance.

At present we do not know how long it would take to stabilize performance at a 1- or 2-sec rate in the History-Dates model. In addition, knowledge of 1,000 instead of 100 historical dates would be required for remembering unconstrained lists of digits. It is also unclear how much additional practice would be needed to move from stable performance at a 5-sec rate to such performance at a 1-sec rate in the Digit-Noun model.

In these case studies, transfer to random, unconstrained digit lists presented at the standard 1-sec rate was not observed. For SP, one reason for this lack of transfer was that we had experimentally constrained his mnemonic system for recoding random digits into meaningful units. BB did have available a complete mnemonic system, but he could not retrieve and re-encode these knowledge elements at the fast rate.

Based on the results from these case studies, we conclude that of the three components Chase and Ericsson (1982) postulated as preconditions of skilled memory, only speed of encoding requires a large amount of practice. It was not difficult to acquire a suitable mnemonic system for recoding digits into meaningful units, and it was not difficult to learn to employ a long-term memory encoding and retrieval structure. Without time constraints on encoding processes, high levels of performance became possible almost instantly after instruction according to the respective models.

What determines improvement in encoding speed? One likely source is speed-up of retrieval of task-relevant facts. Encoding in the models presented here requires the frequent retrieval of knowledge related to landmarks and to digits. There is evidence that fact retrieval improves with practice. For example, Pirolli and Anderson (1985) reported that practice on specific facts and practice on the general task reduced recognition time.

The acquisition of a cognitive expertise requiring well-specified knowledge (i.e., 100 nouns and 40 landmarks in the Digit-Noun model) may permit us to examine the microgenesis of expert knowledge and, in particular, the potentially related increase in encoding and retrieval speed. For example, although we did not collect systematic verbal protocols of our participants' thoughts and images, it was quite clear from informal conversations that, to cope with fast presentation rates, participants changed the mental representation of historical events and landmarks. For example, "crowning of Charlemagne" became "Christmas" because it happened on a 
Christmas day. Similarly, "Devil's Hill" (one of the landmarks) lost its geographic connotation and featured only the "devil" in image generation.

Changes in mental representation as a consequence of selective exposure to task demands are a worthwhile object of study and may play a crucial role in the speed-up and automaticity of performance at high levels. Traditionally, research on experts faced problems of number and comparability of experts. In the present paradigm, synthesizing an expertise in novices according to theoretical principles guarantees comparability at least at a molar level of task organization while still leaving room for individual ingenuity and creativity in the refinement and optimization of task-relevant knowledge.

\section{ACKNOWLEDGMENTS}

This research is part of an ongoing project on "Expertise and Cognitive Aging," co-directed by Paul B. Baltes and Reinhold Kliegl. The authors express their gratitude to Annette Rentz and Werner Scholtysik for expert training and to Wolfgang Assman and Stephan Lempert for the development of computer programs. Helpful comments were provided by Anders Ericsson, Ulman Lindenberger, Tim Salthouse, Alexander von Eye, and Bernd Schmitz. Special thanks go to our two participants (BB and SP) for their interest and persistence.

\section{REFERENCES}

Bellezza, F. S. (1981). Mnemonic devices: Classification, characteristics, and criteria. Review of Educational Research, 51, 247-275.

Bower, G. H. (1970). Analysis of a mnemonic device. American Scientist, 58, 496-510.

Bugelski, B. R. (1970). Words and things and images. American Psychologist, 25, 1002-1012.

Bugelski, B. R., Kidd, E., \& Segmen, J. (1968). The image as a mediator in one-trial paired-associate learning. Journal of Experimental Psychology, 76, 69-73.

Chase, W. G., \& Ericsson, K. A. (1981). Skilled memory. In J. R. Anderson (Ed.), Cognitive skills and their acquisition (pp. 141-189). Hillsdale, NJ: Lawrence Erlbaum Associates, Inc.

Chase, W. G., \& Ericsson, K. A. (1982). Skill and working memory. In G. H. Bower (Ed.), The psychology of learning and motivation (Vol. 16, pp. 1-58). New York: Academic.

Chi, M. T. H., Glaser, R., \& Rees, E. (1982). Expertise in problem solving. In R. J. Sternberg (Ed.), Advances in the psychology of human intelligence (pp. 7-75). Hillsdale, NJ: Lawrence Erlbaum Associates, Inc.

Ericsson, K. A. (1985). Memory skill. Canadian Journal of Psychology, 39, 188-231.

Ericsson, K. A., Chase, W. G., \& Faloon, S. F. (1980). Acquisition of a memory skill. Science, 208, 1181-1182.

Gordon, P., Valentine, E., \& Wilding, J. (1984). One man's memory: A study of a mnemonist. British Journal of Psychology, 75, 1-14.

Hunt, E., \& Love, T. (1972). How good can memory be? In A. W. Melton \& E. Martin (Eds.), 
Coding processes in human memory (pp. 237-260). New York: Winston \& Sons.

James, W. F. (1890). Principles of psychology (Vol. 1). New York: Holt.

Kliegl, R., \& Baltes, P. B. (1987). Theory-guided analysis of mechanisms of development and aging through testing-the-limits and research on expertise. In C. Schooler \& K. W. Schaie (Eds.), Cognitive functioning and social structure over the life course (pp. 95-119). Norwood, NJ: Ablex.

Kliegl, R., Smith, J., \& Baltes, P. B. (1988). Testing-the-limits and the study of age differences in cognitive plasticity of a mnemonic skill. Manuscript submitted for publication.

Lorayne, H., \& Lucas, J. (1974). The memory book. New York: Ballantine.

Luria, A. R. (1968). The mind of a mnemonist. New York: Avon.

Miller, G. A. (1956). The magical number seven, plus or minus two. Psychological Review, 63, 81-97.

Morris, P. E., \& Greer, P. J. (1984). The effectiveness of the phonetic mnemonic system. Human Learning, 3, 137-142.

Neves, D. M., \& Anderson, J. R. (1981). Knowledge compilation: Mechanisms for the automatization of skill. In J. R. Anderson (Ed.), Cognitive skills and their acquisition (pp. 57-84). Hillsdale, NJ: Lawrence Erlbaum Associates, Inc.

Pirolli, P. L., \& Anderson, J. R. (1985). The role of practice in fact retrieval. Journal of Experimental Psychology: Learning, Memory, and Cognition, 11, 136-153.

Simon, H. A. (1986). The parameters of human memory. In F. Klix \& H. Hagendorf (Eds.), Human memory and cognitive capabilities: Mechanisms and performance (pp. 229-309). Amsterdam, Netherlands: North-Holland.

Slak, S. (1970). Phonemic recoding of digital information. Journal of Experimental Psychology, 86, 398-406.

Susukita, T. (1933). Untersuchung eines ausserordentlichen Gedächtnisses in Japan (I) [Examination of an extraordinary memory in Japan (I)]. Tohuku Psychologica Folia, 1, 111-134.

Susukita, T. (1934). Untersuchung eines ausserordentlichen Gedächtnisses in Japan (II) [Examination of an extraordinary memory in Japan (II)]. Tohuku Psychologica Folia, 2, 14-42.

Volkmann, L. (1929). Ars memorativa [The art of memory]. Jahrbuch der Kunsthistorischen Sammlungen in Wien, 3, 111-200.

Yates, F. A. (1966). The art of memory. London: Routledge \& Kegan Paul. 